\title{
New rapid latex agglutination test for diagnosing Trichomonas vaginalis infection
}

\author{
J A CARNEY, * P UNADKAT,$*$ A YULE, $\dagger$ R RAJAKUMAR, $\ddagger$ C J N LACEY, $\dagger$ \\ J P ACKERS $\dagger \quad{ }^{*}$ Mercia Diagnostics Ltd, Guildford, Surrey $\dagger$ Department of Medical Protozoology, London \\ School of Hygiene and Tropical Medicine, and the $\ddagger$ Department of Genitourinary Medicine, General Infirmary, \\ Leeds
}

SUMMARY A newly developed latex agglutination test for Trichomonas vaginalis infection was compared for sensitivity, specificity, efficiency, and positive and negative predictive values with microscopy, culture, and an enzyme linked immunosorbent assay (ELISA) in the diagnosis of 395 women attending a genitourinary medicine clinic. $T$ vaginalis infection was diagnosed in $42(11 \%)$ women. The sensitivities of both the latex agglutination test and the ELISA were $95 \%$ compared with $\mathbf{7 4 \%}$ for microscopy and $76 \%$ for culture. The latex test was specific and showed no cross reaction with a wide range of other genital tract infections.

The latex agglutination test can detect antigen in both soluble and insoluble forms, and as it is simple to perform, can be undertaken during routine examination without recourse to special equipment or training. Further evaluation is required.

Trichomonas vaginalis is a common lower genital tract pathogen. In the United States of America there are an estimated 1.5 million cases of infection each year ${ }^{1}$; 18000 cases of infection were reported from sexually transmitted diseases clinics in the United Kingdom in 1984. ${ }^{2}$ A definitive diagnosis of this infection cannot be made on symptoms and signs alone ${ }^{3}$ as it is commonly associated with other sexually transmitted diseases which may present with similar clinical features. The diagnosis of $T$ vaginalis infection can be confirmed by several methods, including microscopy of wet mounts of vaginal secretions, microscopy of stained vaginal specimens, and culture using various media. ${ }^{4}$ More recently, the techniques of immunofluorescence ${ }^{5}$ and ELISA ${ }^{6}{ }^{7}$ have been developed.

Because of its clinical usefulness, immediate microscopy of fresh vaginal specimens is widely used in diagnosis, but various reports suggest that the diagnostic accuracy of microscopy is only in the range of $56-86 \%^{8-10}$ compared with culture.

We describe and report an evaluation of a new latex slide agglutination test for the diagnosis of $T$ vaginalis infection. This was developed with the aim of producing a rapid, accurate diagnostic tool that might serve

Accepted for publication 26 January 1988 as an alternative to, or improvement on, microscopy in immediate diagnosis.

\section{Patients and methods}

Three hundred and ninety five women attending the department of genitourinary medicine at the General Infirmary, Leeds during the summer of 1986, were studied. A standard history was taken and genital examination performed. Urethral, high vaginal, and endocervical specimens were obtained for examination by both phase contrast $(\times 400)$ and brightfield $(\times 1500)$ oil immersion microscopy. Cultures were taken for Neisseria gonorrhoeae, Chlamydia trachomatis, and Candida albicans as well as for $T$ vaginalis for which a commercially available medium (Oxoid Ltd) was used and the cultures examined after two days.

An additional vaginal swab was obtained from all patients and stored at $-20^{\circ} \mathrm{C}$ for subsequent evaluation by the latex agglutination test. These swabs were tested once without the investigator having knowledge of the other clinical data.

\section{LATEX AGGLUTINATION TEST}

Antiserum was raised in rabbits against axenically cultured whole organisms ${ }^{7}$ and the antibody fraction coated on to polystyrene latex particles (Estapor, 
Rhone-Poulenc) as described previously. ${ }^{11}$ The resultant reagent was tested for sensitivity and consistency of manufacture using a preparation of soluble $T$ vaginalis antigens produced by repeatedly (three times) freeze/thawing the micro-organisms ${ }^{7}$ which had been cultured in modified Diamond's medium. The antibody coating of the test latex reagent was adjusted to give a strong agglutination reaction within two minutes when mixed with this antigen preparation at a concentration of $100 \mu \mathrm{g} / \mathrm{ml}$.

A control latex was produced using non-immune rabbit gamma globulins. ${ }^{11}$

The vaginal swabs were eluted by immersion and agitation in $500 \mu \mathrm{l}$ of glycine buffered saline. The latex test was performed by mixing $30 \mu$ of the eluate with $30 \mu \mathrm{l}$ drops of each of the test and control latexes on a black glass slide. The slide was then rocked continuously for two minutes after which time the results were read. Agglutination of the test but not control latex indicated the presence of $T$ vaginalis antigen. The ELISA, which was performed on all swab eluates, also used the same antibody preparation as was used to coat the latex particles. Dilutions of the soluble antigen solution used as positive control in the latex test were used as standards in the ELISA.

All specimens with soluble antigen concentrations in excess of the lowest standard $(10 \mathrm{ng} / \mathrm{ml})$ were classified as positive.

Patients were considered to have $T$ vaginalis infection when microscopy or culture, or both, were positive. The slide latex agglutination test, the ELISA, microscopy and culture were compared for their sensitivity, specificity, predictive positive value, predictive negative value, and efficiency in the diagnosis of $T$ vaginalis infection.

\section{Results}

Of the 395 women assessed, $42(11 \cdot 1 \%)$ were shown to have $T$ vaginalis infection. The results of the latex agglutination test, ELISA, microscopy and culture are shown in the table. All 42 positive patients had clinical evidence of $T$ vaginalis infection and 21 had one or more additional sexually transmitted infections.

The performance of the latex reagents was clear cut and none of the samples tested caused agglutination of the control latex. Diagnostically, the results of the ELISA were identical with those from the latex tests. The lowest concentration of soluble antigen measured in any clinically positive patients sample was $0.9 \mu \mathrm{g} /$ $\mathrm{ml}$. This specimen was also the slowest in reacting by slide agglutination and required fully two minutes to produce definite aggregation of the particles.

Neither microscopy nor culture were as sensitive as the latex agglutination test or the ELISA in detecting infection. These methods diagnosed $31,32,40$, and 40 cases, respectively, of the total of 42 infected patients. In only two cases did the latex give a false positive result as defined. One of these patients had already received $400 \mathrm{mg}$ of clotrimazole locally after presenting to her family doctor with vulvo-vaginitis. The other presented with vulvo-vaginitis and a purulent vaginal discharge, but microscopy and culture were negative.

Trichomonal infection was suspected clinically and both symptoms and signs resolved with metronidazole. These patients were also positive by ELISA.

\section{Discussion}

Microscopy of wet mounts of vaginal secretions is the only diagnostic technique currently available in the immediate diagnosis of $T$ vaginalis. The acknowledged insensitivity of the test is in part due to the rapid loss of the characteristic motility of the micro-organisms by which they are readily identified. Microscopy, therefore, has to be performed shortly after obtaining vaginal specimens. The recently described immunofluorescence test seems to have improved characteristics compared with conventional microscopy. ${ }^{5}$ Such techniques, however, require at least 30 minutes to perform and the availability of a fluorescence microscope. Newly developed ELISA techniques $^{6} 7$ also seem more sensitive than microscopy but take at least one hour to perform and are essentially laboratory tests.

Table Comparison of latex test, ELISA, microscopy and culture among 395 patients

\begin{tabular}{|c|c|c|c|c|c|c|c|c|}
\hline \multirow[b]{2}{*}{$T$ vaginalis Infection } & \multicolumn{2}{|l|}{ Latex } & \multicolumn{2}{|l|}{$E L I S A$} & \multicolumn{2}{|c|}{ Microscopy } & \multicolumn{2}{|l|}{ Culture } \\
\hline & Positive & Negative & Positive & Negative & Positive & Negative & Positive & Negative \\
\hline $\begin{array}{l}\text { Positive }(n=42) \\
\text { Negative }(n=353) \\
\text { Sensitivity } \\
\text { Specificity } \\
\text { Positive predictive value } \\
\text { Negative predictive value } \\
\text { Efficiency }\end{array}$ & $\begin{array}{rrr}40 & \\
2 & \\
& 95 \cdot 2 \\
& 99 \cdot 4 \\
& 95 \cdot 2 \\
& 99 \cdot 4 \\
& 99 \cdot 0\end{array}$ & $\begin{array}{r}2 \\
351\end{array}$ & $\begin{array}{rrr}40 & \\
2 & \\
& 95 \cdot 2 \\
& 99 \cdot 4 \\
& 95 \cdot 2 \\
& 99 \cdot 4 \\
& 99 \cdot 0\end{array}$ & $\begin{array}{r}2 \\
351\end{array}$ & $\begin{array}{rr}31 & \\
0 & \\
& 73 \cdot 8 \\
& 100 \\
& 100 \\
& 97 \cdot 0 \\
& 97 \cdot 2\end{array}$ & $\begin{array}{r}11 \\
353\end{array}$ & $\begin{array}{rr}32 & \\
0 & \\
& 76 \cdot 2 \\
& 100 \\
& 100 \\
& 97 \cdot 2 \\
& 97 \cdot 5\end{array}$ & $\begin{array}{r}10 \\
353\end{array}$ \\
\hline
\end{tabular}


The rapid latex agglutination test reported here is very similar to one described for the diagnosis of vaginal candidosis, and the clinical trial was conducted in a similar manner. ${ }^{12}$

The evaluation was carried out in a busy sexually transmitted disease clinic with normal clinical and laboratory practice. In this trial the sensitivity of the culture technique was only $76 \%$, and while this is lower than some reports, ${ }^{910}$ it is comparable with others. ${ }^{8}$ Microscopy was almost as sensitive as culture in this study.

In contrast, the sensitivity of the latex agglutination test was higher at $95 \%$ and it seemed to be detecting infections missed by either microscopy or culture. It was also highly specific ( $>99 \%$ ) and was not susceptible to interference from erythrocytes, leucocytes, epithelial cells, normal or pathological vaginal flora. There was stong clinical evidence that one of the two "false" positive results, given by both latex and ELISA was a genuine infection which had been missed by both microscopy and culture. Similar findings have been reported previously in the clinical evaluation of an ELISA for $T$ vaginalis antigen. ${ }^{7}$

There was an exact agreement between results from the slide test and the ELISA. The latex agglutination test, with a lower sensitivity limit of $100 \mu \mathrm{g}$ soluble antigen $/ \mathrm{ml}$, gave positive reactions with several patients' samples found to have much lower soluble antigen concentrations $(0.9 \mu \mathrm{g} / \mathrm{ml})$ by ELISA. While the composition of the soluble antigen preparation may have differed from the antigens presented during infection and consequently have been less immunologically reactive, a more likely explanation is that the bulk of trichomonal antigen is in an insoluble form as intact and partially degraded parasite fragments. Polymorphonuclear leucocytosis is often associated with symptomatic trichomonal infection and may aid in the degradation of the organism. In addition to not requiring the separation of the antibody bound antigen from the rest of the sample, the latex agglutination test may have another important advantage over ELISA in that it can detect antigen in both soluble and insoluble forms.

Although the latex agglutination test was performed in the laboratory, it has been shown that a physician can successfully undertake a test of this type during routine examination of a patient. ${ }^{13}$ Being very simple to perform, this test requires no apparatus nor any training in interpretation of results. The time required to perform this test in any clinical setting is less than five minutes.
Wet mount microscopy and latex agglutination tests are two rapid, simple investigations, the results of which are immediately available to the clinician to confirm the clinical suspicion of trichomonal infection. Although both techniques are highly specific $(>99 \%)$, the latex test has a considerably greater sensitivity $(95 \% v 74 \%)$. We believe this latex agglutination test to be a very useful alternative to conventional diagnostic methods for $T$ vaginalis infection and that it merits further evaluation.

\section{References}

1 US Department of Health and Human Services. Sexually transmitted disease statistics 1983. Centers for Disease Control: Atlanta, Georgia 1985.

2 Communicable Disease Surveillance Centre. Sexually transmitted disease surveillance in Britain: 1984. Communicable Disease Report CDR 86/29. London: 1986.

3 Fouts AC, Kraus SJ. Trichomonas vaginalis: re-evaluation of its clinical presentation and laboratory diagnosis. $J$ Infect Dis 1980;141:137-43.

4 Lowe GH. The Trichomonads. Public Health Laboratory Service Monograph series No 9. London: 1978.

5 Kreiger JN, Holmes KK, Spence MR, McCormack WM, Tam MR. Geographic variation among isolates of Trichomonas vaginalis: demonstration of antigenic heterogeneity by using monoclonal antibodies and the indirect immunofluorescence technique. J Infect Dis 1985;152:979-84.

6 Watt RM, Philip A, Wos SM, Sam GJ. Rapid assay for immunological detection of Trichomonas vaginalis. J Clin Microbiol 1986;24:551-55.

7 Yule A, Gellan MCA, Oriel JD, Ackers JP. Detection of Trichomonas vaginalis antigen in women by enzyme immunoassay. J Clin Pathol 1987;40:566-8.

8 McCann JS. Comparison of direct microscopy and culture in the diagnosis of trichomoniasis. British Journal of Venereal Disease 1974;50:450-2.

9 Ackers JP, Lumsden WHR, Catterall RD, Coyle R. Antitrichomonal antibody in the vaginal secretions of women infected with Trichomonas vaginalis. British Journal of Venereal Disease 1975;51:319-23.

10 Spence MR, Hollander DH, Smith J, McCaig L, Sewell D, Brockman M. The clinical and laboratory diagnosis of Trichomonas vaginalis infection. Sex Transm Dis 1980;7: 168-71.

11 Hopwood V, Evans EGV, Carney JA. Rapid diagnosis of vaginal candiosis by latex particle agglutination. J Clin Pathol 1985; 38:455-8.

12 Evans EGV, Lacey CJN, Carney JA. Criteria for the diagnosis of vaginal candidosis: evaluation of a new latex agglutination test. Eur J Obstet Gynaecol Reprod Biol 22:365-71.

13 Rajakumar R, Lacey CJN, Evans EGC, Carney JA. Use of slide latex agglutination test for rapid diagnosis of vaginal candidosis. Genitourin Med 1987;63:192-5.

Requests for reprints to: Dr J A Carney, Mercia Diagnostics Ltd, Broadford Park, Shalford, Guildford, Surrey GU4 8EW, England. 